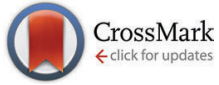

Cite this: Phys. Chem. Chem. Phys., 2015, 17, 16894

Received 20th April 2015, Accepted 1st June 2015

DOI: $10.1039 / \mathrm{c} 5 \mathrm{cp} 02302 \mathrm{e}$

www.rsc.org/pccp

\title{
The effect of external heat transfer on thermal explosion in a spherical vessel with natural convection
}

\begin{abstract}
A. N. Campbell
When any exothermic reaction proceeds in an unstirred vessel, natural convection may develop. This flow can significantly alter the heat transfer from the reacting fluid to the environment and hence alter the balance between heat generation and heat loss, which determines whether or not the system will explode. Previous studies of the effects of natural convection on thermal explosion have considered reactors where the temperature of the wall of the reactor is held constant. This implies that there is infinitely fast heat transfer between the wall of the vessel and the surrounding environment. In reality, there will be heat transfer resistances associated with conduction through the wall of the reactor and from the wall to the environment. The existence of these additional heat transfer resistances may alter the rate of heat transfer from the hot region of the reactor to the environment and hence the stability of the reaction. This work presents an initial numerical study of thermal explosion in a spherical reactor under the influence of natural convection and external heat transfer, which neglects the effects of consumption of reactant. Simulations were performed to examine the changing behaviour of the system as the intensity of convection and the importance of external heat transfer were varied. It was shown that the temporal development of the maximum temperature in the reactor was qualitatively similar as the Rayleigh and Biot numbers were varied. Importantly, the maximum temperature in a stable system was shown to vary with Biot number. This has important consequences for the definitions used for thermal explosion in systems with significant reactant consumption. Additionally, regions of parameter space where explosions occurred were identified. It was shown that reducing the Biot number increases the likelihood of explosion and reduces the stabilising effect of natural convection. Finally, the results of the simulations were shown to compare favourably with analytical predictions in the classical limits of Semenov and Frank-Kamenetskii.
\end{abstract}

\section{Introduction}

When any exothermic reaction occurs in a closed vessel then there is a risk of thermal explosion. Such explosions occur when the rate of heat production by the reaction exceeds the rate of heat removal through the vessel's walls. The pioneering works on thermal explosion by Semenov ${ }^{1}$ and Frank-Kamenetskii ${ }^{2}$ considered two extreme cases. Semenov investigated cases where the mixing within the vessel was so efficient that its contents could be considered to be spatially uniform. He proposed a dimensionless group, which has come to be known as the Semenov number,

$$
\psi=\frac{k_{0} c_{0}^{n} q E}{h S_{\mathrm{v}} R T_{0}^{2}}
$$

Department of Chemical and Process Engineering, Faculty of Engineering and Physical Sciences, University of Surrey, Guildford GU2 7XH, UK.

E-mail: a.n.campbell@surrey.ac.uk; Tel: +44(0)1483682130
Semenov showed that an explosion would occur when $\psi$ exceeds the critical value of $1 / e$. In contrast, Frank-Kamenetskii considered a case without mixing. In such a scenario, spatial temperature gradients develop. Frank-Kamenetskii realised that these gradients would significantly impact the heat removal from the system, and hence the tendency to explode. In particular, he considered cases where heat transfer occurred by conduction only. Frank-Kamenetskii defined the parameter $\delta=L^{2} k_{0} c_{0}^{n} q E /$ $\kappa \rho_{0} C_{\mathrm{P}} R T_{0}{ }^{2}$ and showed that explosion occurred when $\delta$ exceeded a critical value, which was dependent on the geometry.

If the temperature gradients become sufficiently large, the resulting buoyancy forces will cause the gas to move. This natural convection can have a significant influence on the progress of the reaction. The nature of the induced flow is determined by the Rayleigh number, $\mathrm{Ra}=\left(\beta g L^{3} \Delta T\right) /(\kappa \nu)$. Frank-Kamenetskii ${ }^{2}$ suggested that in a gaseous system, natural convection becomes significant when $\mathrm{Ra}>10^{4}$; however, further studies ${ }^{3-5}$ have shown that natural convection becomes important when Ra exceeds $\sim 500$. 
The initial experimental evidence ${ }^{3}$ for a greater influence of natural convection than was allowed for by Frank-Kamenetskii in his model inspired a number of experimental and numerical investigations of exothermic reactions with natural convection. The experimental work of Merzhanov and Shtessel ${ }^{6}$ on liquid systems in a horizontal cylinder showed that natural convection is significant at Rayleigh numbers well below $10^{4}$. These empirical observations of the effects of natural convection at lower values of $\mathrm{Ra}$ are supported by the analytical and numerical work of Jones ${ }^{7}$ and Shtessel et al.; ${ }^{8}$ they showed that when a zeroth-order reaction (i.e. one in which the depletion of the reactant is ignored) occurs between parallel plates, the critical Rayleigh number for the onset of convection is again $\sim 500$. Jones ${ }^{7}$ also noted that inside a horizontal cylinder or a sphere, there is always some convection, because of the existence of temperature gradients perpendicular to the gravity vector; the 'onset' of convection is in fact merely the point where the effects of this flow become observable. In a seminal work on the interaction of thermal explosion and natural convection, Merzhanov and Shtessel ${ }^{9}$ presented a detailed numerical and experimental analysis of explosion in a liquid system. They showed that natural convection suppresses explosion, and that when explosion does occur, the ignition delay is longer in the presence of natural convection.

In all of these numerical studies, the effect of Ra on thermal explosion was quantified by calculating a new critical value of the Frank-Kamenetskii number, $\delta$. More recently, a new approach was proposed by Liu et al. ${ }^{10}$ who presented an analysis in which the explosive limits were described in terms of a series of ratios of characteristic timescales for the important physical processes that are at work, namely natural convection, diffusion (of both heat and mass) and heating due to the chemical reaction. This approach is very attractive, as it describes the transition to explosion in terms of simple groups, which have clear physical meaning. Liu et $a .^{10}$ summarised their results using a new regime diagram, where the ordinate is the ratio of the timescales for heating and diffusion and the abscissa is the ratio of the timescales for heating and convection. Liu et al. ${ }^{10}$ also presented clear criteria whereby the effect of consumption of reactant on the progress of an explosion could be ignored. Thus, they identified regions of parameter space when explosions occurred without ${ }^{10}$ and subsequently with ${ }^{11}$ reactant consumption included. Significantly, Liu et al. ${ }^{11}$ also revisited the issue of defining when an explosion can be deemed to have occurred in a system with significant depletion of reactant. This transition to explosion is obvious in systems with minimal reactant consumption, where in an explosive case the temperature will increase without bound; however, when the reactant is depleted, this will significantly reduce the production of heat by the reaction. Consequently, a plot of the temporal development of the maximum temperature in the vessel will display a clear maximum. Liu et al. ${ }^{11}$ proposed a simple criterion to determine whether an explosion has occurred based solely on the maximum dimensionless temperature achieved. When this exceeds 5, an explosion is said to have occurred. This simple criterion was also shown to be applicable in cases with more complex chemical reactions occuring, ${ }^{12,13}$ and in reactors where both natural and forced convection are important. ${ }^{14,15}$
Many other numerical studies have considered the effect of natural convection on explosion in so-called class A geometries (i.e. parallel plates, infinite cylinders and spheres). A comprehensive listing can be found elsewhere. ${ }^{10}$ These studies, however, all consider cases in which the fixed boundaries of the reactor are held at a constant temperature. This constant temperature at the wall implies there is an infinite heat transfer coefficient between the reactor and its surroundings. In reality, this is unlikely to be the case. There will, of course, be a heat transfer resistance associated with conduction through the solid wall of the reactor, and a finite resistance between the wall and the environment. It is obvious that these additional heat transfer mechanisms may significantly alter the rate of heat removal from the reactor, and hence the tendency of the fluid to explode. Despite this important fact, very few studies have included the effect of external heat transfer in their analyses. Frank-Kamenetskii ${ }^{2}$ did derive an approximate expression for the dependence of $\delta$ in a purely diffusive system on external heat transfer, via what he called a Nusselt number, but what is in reality a Biot number. He found that the inclusion of external heat transfer made an explosion more likely. Boddington et al. ${ }^{16}$ performed an elegant asymptotic analysis of a purely diffusive system in which consumption of reactant is important. They showed that external heat transfer, again characterised by a Biot number, had a more significant effect in a spherical system, when compared with the other class A geometries.

This work aims to perform an initial investigation into the transition from a slow reaction to explosion in a spherical reactor in which natural convection is significant. As will be justified below, the depletion of the reactant will be ignored. The governing equations are presented in Section 2. The results of numerical simulations are discussed in Section 4. In addition, the results are compared with the analytical results of Semenov and Frank-Kamenetskii.

\section{Theoretical background}

\subsection{Dimensional equations}

The reaction occurring in a spherical reactor is considered to be of the form

$$
\mathrm{A} \rightarrow \text { products }+ \text { heat }
$$

i.e. it is considered to be an $n$th order exothermic, thermal decomposition reaction. The governing equations express the conservation of the reactant, energy, momentum and mass. The Boussinesq approximation, in which density changes are ignored in all terms except the body forces (which drive natural convection) in the Navier-Stokes equations, is applied to considerably simplify the equations. The Boussinesq approximation requires that the characteristic temperature rise $\Delta T$ is such that $\Delta T \ll T_{0}$; otherwise, full compressibility needs to be taken into account. This approximation is commonly used in the analysis of buoyant flows. ${ }^{17}$ This approach has been used extensively in the study of thermal explosion, ${ }^{6-15,18-20}$ as well as 
other exothermic reactions, ${ }^{21-28}$ under the influence of natural convection.

The equation governing the conservation of the reactant can be written as:

$$
\frac{\partial c}{\partial t}+\underline{u} \cdot \nabla c=D_{\mathrm{C}} \nabla^{2} c-k(T) c^{n}
$$

where $c$ is the concentration of the reactant, $\underline{u}$ is the velocity vector, $D_{\mathrm{C}}$ is the molecular diffusivity and $n$ is the order of the reaction. The rate constant $k$ is assumed to have Arrhenius temperature dependence, i.e. it has the form $k=Z \exp (-E / R T)$, where $Z$ is the pre-exponential factor and $E$ is the activation energy. It should be noted that the diffusion is assumed to be Fickian, i.e. the diffusive flux is proportional to concentration gradients, as opposed to gradients in mole fraction. This is a consequence of the adoption of the Boussinesq approximation. The conservation of energy within the reactor is described by:

$$
\frac{\partial T}{\partial t}+\underline{u} \cdot \nabla T=\kappa \nabla^{2} T+\frac{q k(T)}{\rho_{0} C_{\mathrm{P}}} c^{n},
$$

where $C_{\mathrm{P}}$ is the mixture's specific heat at constant pressure, $T$ is its temperature, $\kappa$ is the thermal diffusivity, $\rho_{0}$ is the density at the initial temperature $T_{0}$ and $q$ is the exothermicity of the reaction. The familiar Navier-Stokes equations:

$$
\frac{\partial \underline{u}}{\partial t}+\underline{u} \cdot \nabla \underline{u}=-\frac{1}{\rho_{0}} \nabla\left(P-P_{0}\right)+\nu \nabla^{2} \underline{u}+\frac{\rho-\rho_{0}}{\rho_{0}} \underline{g},
$$

describe conservation of momentum, where $P$ is the pressure in the reactor and $\nu$ is the kinematic viscosity. Per the Boussinesq approximation, the density only varies in the buoyancy term of eqn (5), in which $\rho=\rho_{0}\left[1-\beta\left(T-T_{0}\right)\right]$, where $\beta$ is the coefficient of thermal expansion.

The final equation required is the continuity equation. Adoption of the Boussinesq approximation allows the continuity equation to be written in its incompressible form, i.e.

$$
\nabla \cdot \underline{u}=0
$$

Initially, the reactor is considered to contain the reactant at a concentration $c_{0}$, and the contents are assumed to be well-mixed. The gas is also assumed to be initially motionless and at a uniform temperature $T_{0}$; this is the temperature of the surrounding environment, which remains fixed throughout the course of the reaction. The external resistance to heat transfer is modelled via a heat transfer coefficient. This condition, of course, means that the temperature of the wall will not remain constant over the duration of the simulation. It is assumed that the no-slip condition applies at the wall and that there is no flux of any species at the wall. The effects of any heterogeneous reactions at the wall have hence been ignored. The initial and boundary conditions can thus be stated:

$$
t=0: \quad c=c_{0} ; T=T_{0} ; \underline{u}=0 \quad \forall \underline{x}
$$

At the wall: $\underline{n} \cdot \nabla c=\underline{u}=0 ; \underline{n} \cdot\left(-k_{\mathrm{T}} \nabla T\right)=h\left(T-T_{0}\right)$,

where $\underline{n}$ is a unit vector perpendicular to the wall of the vessel.

\subsection{Dimensionless equations}

Following the method proposed by Liu et al. ${ }^{10}$ the governing eqn (3)-(7) can be made dimensionless by the introduction of the following dimensionless variables:

$c=\frac{c}{c_{0}} ; T^{\prime}=\frac{T-T_{0}}{\Delta T} ; \underline{u^{\prime}}=\frac{\underline{u}}{U} ; P^{\prime}=\frac{P-P_{0}}{\rho_{0} U^{2}} ; \underline{x^{\prime}}=\frac{\underline{x}}{L}$ and $t^{\prime}=\frac{U t}{L}$,

where $\Delta T=R T_{0}{ }^{2} / E$ is a characteristic scale for the rise in temperature due to the reaction, $U=(\beta g L \Delta T)^{1 / 2}$ is a characteristic velocity when natural convection dominates transport and $L$ is the characteristic length scale for the reactor, taken to be the radius in the present work. Additionally, the following characteristic timescales can be defined:

$$
\tau_{\mathrm{D}}=\frac{L^{2}}{\kappa} ; \tau_{\mathrm{C}}=\frac{L}{U} \text { and } \tau_{\mathrm{H}}=\frac{\rho_{0} C_{\mathrm{P}} R T_{0}^{2}}{k_{0} c_{0}^{n} q E},
$$

for diffusion, natural convection and heating due to the reaction, respectively. Here, $k_{0}$ is the rate constant evaluated the initial temperature $T_{0}$. Eqn (8) and (9) allow the governing eqn (3)-(7) to be written as dimensionless form as:

$$
\begin{gathered}
\frac{\partial c^{\prime}}{\partial t^{\prime}}+\underline{u^{\prime}} \cdot \nabla^{\prime} c^{\prime}=\frac{1}{\operatorname{Le}} \frac{\tau_{\mathrm{C}}}{\tau_{\mathrm{D}}} \nabla^{\prime 2} c^{\prime}-\frac{1}{T_{\mathrm{ad}}} \frac{\tau_{\mathrm{C}}}{\tau_{\mathrm{H}}} \exp \left(\frac{T^{\prime}}{1+\eta T^{\prime}}\right) c^{\prime n}, \\
\frac{\partial T^{\prime}}{\partial t^{\prime}}+\underline{u^{\prime}} \cdot \nabla^{\prime} T^{\prime}=\frac{\tau_{\mathrm{C}}}{\tau_{\mathrm{D}}} \nabla^{\prime 2} T^{\prime}+\frac{\tau_{\mathrm{C}}}{\tau_{\mathrm{H}}} \exp \left(\frac{T^{\prime}}{1+\eta T^{\prime}}\right) c^{\prime n}, \\
\frac{\partial \underline{u^{\prime}}}{\partial t^{\prime}}+\underline{u^{\prime}} \cdot \nabla^{\prime} \underline{u^{\prime}}=-\nabla^{\prime} P^{\prime}+\operatorname{Pr} \frac{\tau_{\mathrm{C}}}{\tau_{\mathrm{D}}} \nabla^{\prime 2} \underline{u^{\prime}}-\frac{g}{=} T^{\prime}, \\
\nabla^{\prime} \cdot \underline{u^{\prime}}=0, \\
t^{\prime}=0: \quad c^{\prime}=1 ; T^{\prime}=0 ; \underline{u^{\prime}}=0 \quad \forall \underline{x^{\prime}} \\
\text { At the wall: } \underline{n} \cdot \nabla^{\prime} c^{\prime}=\underline{u}^{\prime}=0 ;-\underline{n} \cdot \nabla^{\prime} T^{\prime}=\mathrm{Bi} T^{\prime},
\end{gathered}
$$

where Le $=\kappa / D_{\mathrm{C}}$ is the Lewis number, $T_{\mathrm{ad}}{ }^{\prime}=q c_{0} E /\left(\rho_{0} C_{\mathrm{P}} R T_{0}{ }^{2}\right), \eta=$ $R T_{0} / E, \operatorname{Pr}=\nu / \kappa$ is the Prandtl number and $\mathrm{Bi}=h L / k_{\mathrm{T}}$ is the Biot number. It should be noted that the form of $\mathrm{Bi}$ is slightly different to the classical definition, which uses the ratio of volume and surface area as the characteristic length scale. ${ }^{29}$ For a spherical reactor this length is $L / 3$, hence the form in eqn (14) only differs from the classical definition by a factor of 3 . The limiting cases of constant wall temperature and perfect mixing are represented by $\mathrm{Bi}=\infty$ and $\mathrm{Bi}=0$, respectively. The governing dimensionless eqn (10)-(13) are the same as those presented previously, ${ }^{10}$ whilst the boundary conditions in eqn (14) differ.

The following criteria were proposed, ${ }^{10}$ based on the equations above, to determine whether the consumption of reactant is significant. Specifically, if

$$
\frac{\tau_{\mathrm{H}}}{\tau_{\mathrm{C}}} \gg \frac{1}{T_{\mathrm{ad}}{ }^{\prime}} \text { and } \frac{\tau_{\mathrm{H}}}{\tau_{\mathrm{D}}} \gg \frac{\text { Le }}{T_{\mathrm{ad}}{ }^{\prime}}
$$

then it may be assumed that the concentration of the reactant is approximately constant throughout the course of the reaction and eqn (10) can be ignored. For the purposes of this initial 


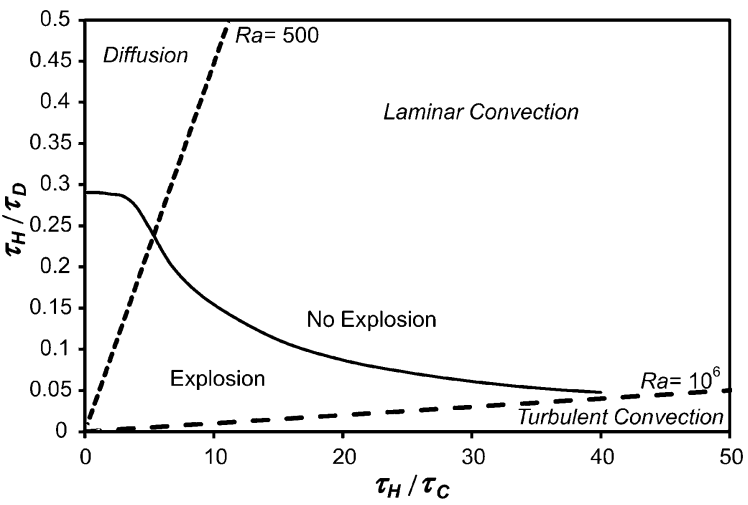

Fig. 1 The explosion diagram proposed by Liu et al. ${ }^{10}$ Plotted on the ordinate is the ratio of the characteristic timescales for heating by the reaction and the diffusion of heat and on the abscissa, the ratio of the timescales for heating and convection. The dashed lines represent constant values of Ra $\left(500\right.$ and $\left.10^{6}\right)$ and separate the parameter space into regions where diffusion, laminar natural convection and turbulent natural convection dominate the transport of heat and mass. The solid black line represents the boundary between the stable and explosive regions for $\mathrm{Bi}=\infty$ (i.e. when the temperature of the wall remains constant) in a spherical vessel. The explosive boundary was found by performing many simulations and agrees with that found previously. ${ }^{10}$

study, this is assumed to be the case. Thus, only the equations for the conservation of energy, momentum and mass must be solved. The behaviour of this system can therefore be characterised by the following six dimensionless groups:

$$
\frac{\tau_{\mathrm{H}}}{\tau_{\mathrm{D}}}, \frac{\tau_{\mathrm{H}}}{\tau_{\mathrm{C}}}, \eta, \operatorname{Pr}, n, \mathrm{Bi}
$$

and for a given chemical system, where $\eta$, Pr and $n$ are fixed, then the behaviour of the system is governed by the ratios $\tau_{H} / \tau_{D}$ and $\tau_{\mathrm{H}} / \tau_{\mathrm{C}}$, as well as $\mathrm{Bi}$. For a system with a constant wall temperature, the behaviour is only a function of the ratios of timescales as Bi would be infinite. This led to the proposal ${ }^{10}$ of the regime diagram, such as shown in Fig. 1, to describe the system. The vertical axis represents the purely conductive limit, i.e. that considered by Frank-Kamenetskii. Lines of constant Ra can be drawn by recognising that

$$
\mathrm{Ra}=\frac{\beta g L^{3} \Delta T}{\kappa \nu}=\left(\frac{\tau_{\mathrm{D}}}{\tau_{\mathrm{C}}}\right)^{2} \operatorname{Pr}^{-1}=\left(\frac{\tau_{\mathrm{H}}}{\tau_{\mathrm{C}}}\right)^{2}\left(\frac{\tau_{\mathrm{H}}}{\tau_{\mathrm{D}}}\right)^{-2} \operatorname{Pr}^{-1}
$$

and hence straight lines through the origin in Fig. 1 will have constant Ra. Shown in Fig. 1 are lines for $\mathrm{Ra}=500$ and $10^{6}$. For $\mathrm{Ra}<500$ diffusion is the dominant transport mechanism, for $500<\mathrm{Ra}<10^{6}$ laminar natural convection is present and for $\mathrm{Ra}>10^{6}$ the convection is turbulent.

\section{Numerical method}

The governing eqn (4)-(7) were solved using COMSOL Multiphysics $^{\circledR} 4.3 \mathrm{a},{ }^{30}$ which utilises the finite element method. The solution was assumed to be axisymmetric about the vertical axis of the spherical vessel. Accordingly, the equations were solved on a semi-circular, 2-dimensional mesh consisting of approximately 3000 linear triangular elements. The algorithm was tested through comparison with previous numerical studies of single ${ }^{10,11,19}$ and two-step ${ }^{23-26}$ exothermic reactions. The algorithm used in these previous studies was verified through comparison with analytical solutions ${ }^{31}$ and experimental results. ${ }^{19}$ Indeed, the explosive limit for a constant wall temperature (i.e. $\mathrm{Bi}=\infty$ ) shown in Fig. 1 is consistent with that found by Liu et al. ${ }^{10}$ in their numerical study, which they showed agreed well with experimental measurements.

For the numerical simulations the thermal decomposition of azomethane was considered. The order of this reaction has been found experimentally as $n=1.4 .^{5}$ The physico-chemical properties employed were: $C_{\mathrm{p}}=2250 \mathrm{~J} \mathrm{~kg}^{-1} \mathrm{~K}^{-1}, Z=1.24 \times$ $10^{14} \mathrm{~mol}^{-0.4} \mathrm{~m}^{1.2} \mathrm{~s}^{-1}, E / R=23280 \mathrm{~K}\left(\right.$ so $E=193.6 \mathrm{~kJ} \mathrm{~mol}^{-1}$ ). The exothermicity of the reaction was assumed to be $q=$ $124 \mathrm{~kJ} \mathrm{~mol}^{-1}$. In accordance with previous studies, the radius of the reactor was taken to be $L=0.064 \mathrm{~m}$, corresponding to a reactor with a volume of $1100 \mathrm{dm}^{3}$. The temperature of the environment was taken as $T_{0}=636.2 \mathrm{~K}$, which matches the wall temperature used in previous studies. ${ }^{10,11}$ The concentration of azomethane in the reactor was assumed to remain constant, as discussed above, with $c_{0}=0.37 \mathrm{~mol} \mathrm{~m}^{-3}$. The ratios $\tau_{\mathrm{H}} / \tau_{\mathrm{D}}$ and $\tau_{\mathrm{H}} / \tau_{\mathrm{C}}$ were varied by changing the value of $\kappa$ and $g$, respectively. The external heat transfer coefficient $h$ was then varied to give the desired Bi. Simulations were performed for $\mathrm{Ra}<10^{6}$, i.e. cases where the natural convection was laminar. The system was further simplified by assuming that $\operatorname{Pr}=1$, i.e. the diffusivities of heat and momentum were assumed to be equal. This is approximately true for gasses but not for liquids.

\section{Results and discussion}

\subsection{Temporal and spatial development of the temperature and speed}

The inclusion of an external heat transfer resistance alters the heat removal from the system. Additionally, the temperature of the wall is no longer constant throughout the duration of the reaction. Both of these factors may alter the way the reaction proceeds, and hence the spatial structures within the reactor. To investigate this difference, eighteen simulations were run to examine the difference between stable and explosive reactions at three different values of $\mathrm{Bi}$. The values considered are $\mathrm{Bi}=10$ (where external heat transfer is significant, but internal resistances still dominate overall), $\mathrm{Bi}=1$ (where internal and external thermal resistances are similar in magnitude) and $\mathrm{Bi}=0.1$ (where external heat transfer is the dominant mechanism). Additionally, three intensities of natural convection, as characterised by $\mathrm{Ra}$ (calculated via eqn (17)) were considered: $\mathrm{Ra}=10^{3}$, where natural convection is becoming important, $\mathrm{Ra}=10^{4}$, where laminar convection is dominant and $\mathrm{Ra}=10^{5}$, where the system is approaching the transition to turbulence. The conditions for the eighteen cases (A-R) are summarised in Table 1. Plotted in Fig. 2 are the temporal developments of the maximum dimensionless temperatures in the reactor for each of cases A-R. For $\mathrm{Bi}=10$, it is clear that the temporal development of the stable cases (A, C and E) is remarkably similar. In each case, the 
Table 1 The eighteen cases considered in Fig. 2 and 3. The constant physico-chemical parameters used appear in Section 3. The dimensionless groups $\tau_{\mathrm{H}} / \tau_{\mathrm{C}}$ and $\tau_{\mathrm{H}} / \tau_{\mathrm{D}}$, which locate a point on the regime diagram in Fig. 1, were varied by changing $g$ and $\kappa$ respectively. The values of these ratios were chosen to give $\mathrm{Ra}=10^{3}, 10^{4}$ or $10^{5}$, as found using eqn (17). The Biot number was chosen by varying $h$. The cases A-R are separated into stable reactions (S) and explosive reactions (E)

\begin{tabular}{|c|c|c|c|c|c|}
\hline Case & $\frac{\tau_{\mathrm{H}}}{\tau_{\mathrm{C}}}$ & $\frac{\tau_{\mathrm{H}}}{\tau_{\mathrm{D}}}$ & $\mathrm{Bi}$ & $\mathrm{Ra}$ & State \\
\hline A & 9.5 & 0.300 & \multirow[t]{6}{*}{10} & $10^{3}$ & $\mathrm{~S}$ \\
\hline B & 8.7 & 0.275 & & & E \\
\hline $\mathrm{C}$ & 22.7 & 0.227 & & $10^{4}$ & $\mathrm{~S}$ \\
\hline D & 20.5 & 0.205 & & & E \\
\hline $\mathrm{E}$ & 55 & 0.174 & & $10^{5}$ & $\mathrm{~S}$ \\
\hline $\mathrm{F}$ & 50 & 0.158 & & & E \\
\hline G & 34.5 & 1.09 & \multirow[t]{6}{*}{1} & $10^{3}$ & $\mathrm{~S}$ \\
\hline $\mathrm{H}$ & 32.5 & 1.02 & & & E \\
\hline I & 106 & 1.06 & & $10^{4}$ & $\mathrm{~S}$ \\
\hline $\mathrm{J}$ & 98 & 0.98 & & & $\mathrm{E}$ \\
\hline K & 325 & 1.03 & & $10^{5}$ & S \\
\hline $\mathrm{L}$ & 300 & 0.949 & & & $\mathrm{E}$ \\
\hline M & 290 & 9.17 & \multirow[t]{6}{*}{0.1} & $10^{3}$ & $\mathrm{~S}$ \\
\hline $\mathrm{N}$ & 275 & 8.69 & & & E \\
\hline $\mathrm{O}$ & 915 & 9.15 & & $10^{4}$ & $\mathrm{~S}$ \\
\hline $\mathrm{P}$ & 870 & 8.7 & & & E \\
\hline Q & 2900 & 9.17 & & $10^{5}$ & $S$ \\
\hline $\mathrm{R}$ & 2750 & 8.70 & & & $\mathrm{E}$ \\
\hline
\end{tabular}

temperature rises at a relatively constant rate initially, before the rate of increase slows as the natural convection develops and enhances heat removal. When convection becomes fully established, the maximum temperature reaches its steady value. In all three cases the maximum value at steady state is $T^{\prime} \sim 1.5$. The explosive cases (B, D and F) show a similar rise in temperature to the non-explosive cases initially. Unsurprisingly, after this initial period, the maximum temperature in the vessel is higher than is the case for the stable reactions. In all three cases there is clearly a decrease in the slope of the temperature versus time curve (i.e. $\partial^{2} T^{\prime} / \partial t^{\prime 2}<0$ ) before an upward inflection in the curve, after which the temperature increases without bound. Such a shape of curve is, of course, characteristic of an explosive system in which reactant consumption is ignored. If consumption of reactant was important, then the increase in temperature would remain bounded, due to the limited heat production by the reaction of a finite mass. In each case, the inflection in temperature can be seen to occur when $T^{\prime}$ is in the range $1.5-2$. It has been noted previously ${ }^{10}$ that this upward inflection in temperature, which is often used to define whether or not an explosion has occurred, can be seen in nonexplosive cases when natural convection is important. In particular, it is characteristic of cases where significant reaction occurs before natural convection has been established, and once the flow is significant, the heat removal at the wall increases and thus stabilises the reaction. It is worth pointing out, however, that the observation of such non-explosive inflections occurred in the temperature histories of fixed points in the reactor rather than the overall maximum plotted here. It may be that this
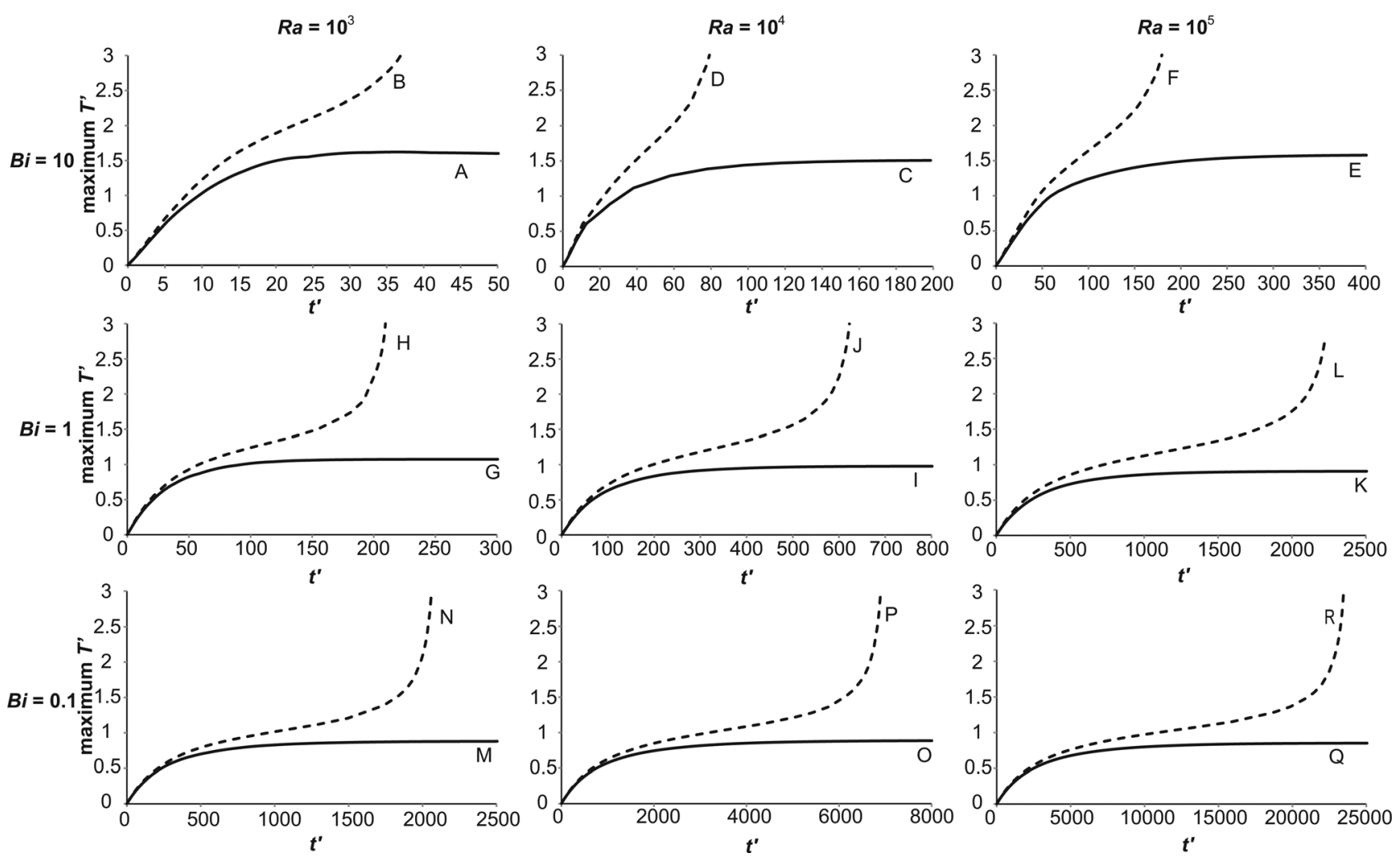

Fig. 2 The temporal evolution of the maximum dimensionless temperature in the reactor for cases A-R. These cases fall either side, but close to, the explosion boundary. The stable reactions are plotted as solid lines, whilst the explosive cases are dashed lines. The plots are ordered such that $\mathrm{Bi}$ is constant along a row and $\mathrm{Ra}$ is constant along a column. The three values of Bi considered are 10,1 and 0.1, whilst the three values of Ra are $10^{3}, 10^{4}$ and $10^{5}$. 
procedure, which allows for the migration of the location of the maximum temperature as the reaction proceeds, means that such non-explosive inflections are not seen. It is also interesting to note that the dimensionless time to explosion increases with increasing Ra. An elegant analysis of localised explosion and convection, which should be independent of geometry and boundary conditions, has been preformed, ${ }^{18}$ and analytical expressions for the transitions between convective and non-convective explosive cases, and the time for the onset of convection developed; however, the time for onset of convection and explosion, and comparison with the analytical expressions developed have not been considered further here.

Similar trends are evident when comparing the stable and explosive cases for reduced values of $\mathrm{Bi}$. For $\mathrm{Bi}=1$, the shapes of the stable cases ( $\mathrm{G}, \mathrm{I}$ and $\mathrm{K}$ ) and the explosive cases ( $\mathrm{H}, \mathrm{J}$ and $\mathrm{L}$ ) are very similar for the three values of $\mathrm{Ra}$, with the only obvious quantitative difference being the time taken to reach the stable steady state or the upward inflection which indicates that the system is proceeding to explosion. The time for both increases with increasing $\mathrm{Ra}$ as was the case for $\mathrm{Bi}=10$. When compared with the cases for $\mathrm{Bi}=10$, it is evident that the temperatures in cases G-L are lower than those in A-F. Specifically, the upward inflection in the temperature occurs at $T^{\prime} \sim 1.5$ for cases $\mathrm{H}, \mathrm{J}$ and $\mathrm{L}$ and the maximum temperature in the stable cases $\mathrm{G}, \mathrm{I}$ and $\mathrm{K}$ is $T^{\prime} \sim 1$. This makes intuitive sense as the increasing levels of external resistance to heat transfer as Bi decreases mean that the ability to remove heat from the system is reduced and hence, the reaction will run away at lower temperatures. Additionally, comparison of cases A-F and G-L indicates that the dimensionless timescales for the onset of explosion or the establishment of the steady state are increased as $\mathrm{Bi}$ is decreased.

When $\mathrm{Bi}=0.1$, i.e. when the heat transfer is largely controlled by the external resistance, the behaviours seen in the stable (M, O and $\mathrm{Q}$ ) and explosive ( $\mathrm{N}, \mathrm{P}$ and $\mathrm{R}$ ) cases are again similar to those described above. The temperature at which the upward inflection is seen is lower than in those cases with higher $\mathrm{Bi}$ and the maximum temperature of the stable systems is once again $T^{\prime} \sim 1$. Therefore, comparison of all nine explosive cases and all nine stable cases reveals that the evolution of the maximum temperature in the vessel is qualitatively similar across the range of $\mathrm{Bi}$ and $\mathrm{Ra}$ considered. The most obvious quantitative difference is that the temperatures seen, both at the point where the explosive reaction accelerates to infinity and in the maximum temperature of the stable system, are smaller as $\mathrm{Bi}$ is increased, but remarkably similar as $\mathrm{Ra}$ is varied at fixed $\mathrm{Bi}$.

It is clear from Fig. 2 that temporal development of the maximum temperature in the vessel is affected very little by either Bi or Ra, with the primary quantitative difference being in the magnitude of the temperatures observed. It is, however, to be expected that the spatial development of the temperature, and hence velocity fields will be more significantly altered by the existence of external heat transfer. Plotted in Fig. 3 are the spatial variations of the temperature and speed of the flow as explosive reactions proceed. Specifically, cases B, F, H, L, N and $\mathrm{R}$ are presented, i.e. the explosive cases for $\mathrm{Bi}=10,1$ and 0.1 with $\mathrm{Ra}=10^{3}$ and $10^{5}$.

Fig. 3(a) shows case $\mathrm{B}$, which has $\mathrm{Bi}=10$ and $\mathrm{Ra}=10^{3}$. It is clear that the system heats up due to the reaction and a hot zone begins to form above the centre of the reactor at $t=2 \mathrm{~s}$. In this case there is weak natural convection and this is what displaces the hot zone towards the top of the reactor. As the gas heats up, its density decreases and the higher temperature, lower density gas near the centre of the reactor experiences buoyancy forces which cause it to move vertically upwards. This high speed zone at the centre of the reactor is evident for $t>2 \mathrm{~s}$. As the hot gas moves vertically upwards it contacts the cold wall and hence begins to descend, cooling further as it goes. This moderately high speed gas descending near the wall is also evident in Fig. 3(a). In the bottom half of the reactor, the density gradients are intrinsically stable and so the buoyancy

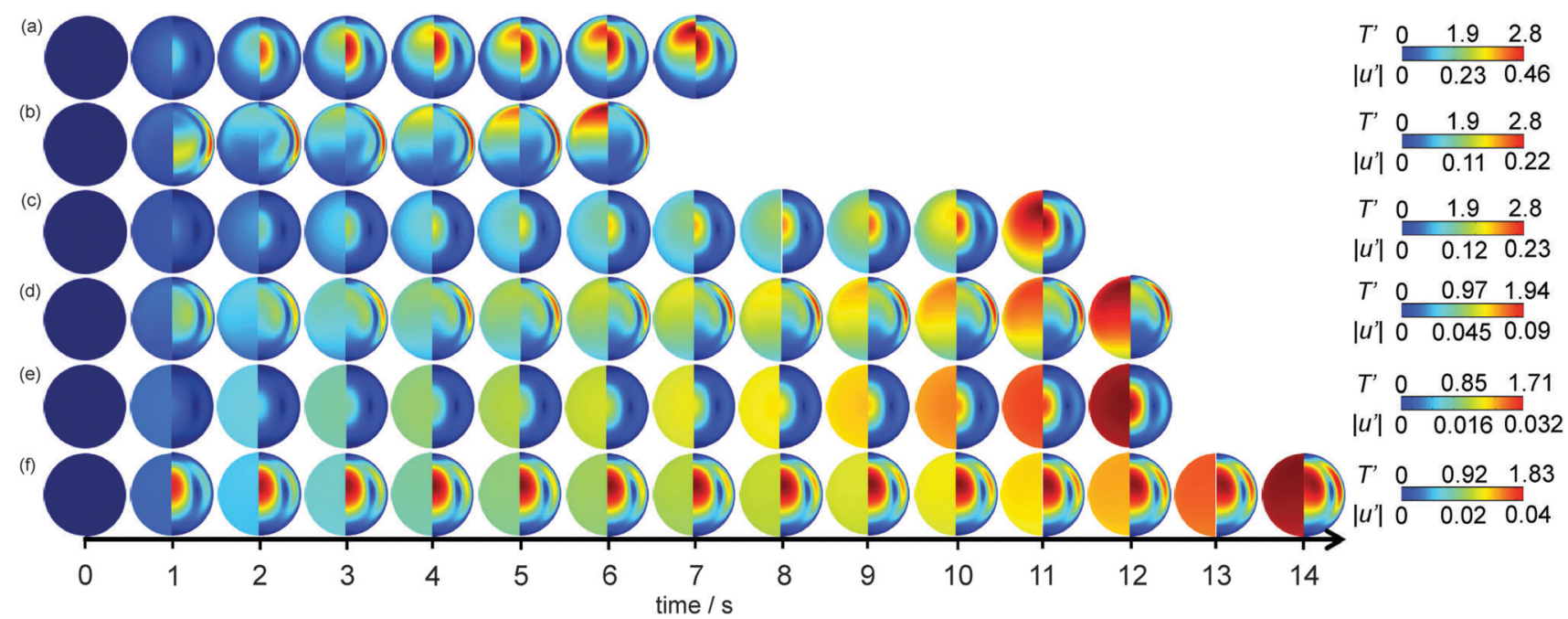

Fig. 3 The temporal development of the spatial dimensionless temperature (left semi-circle) and speed (right semi-circle) profiles in the reactor for (a) case $B\left(B i=10, R a=10^{3}\right)$, (b) case $F\left(B i=10, R a=10^{5}\right)$, (c) case $H\left(B i=1, R a=10^{3}\right)$, (d) case $L\left(B i=1, R a=10^{5}\right)$, (e) case $N\left(B i=0.1, R a=10^{3}\right)$ and $(f) c a s e ~ R$ $\left(\mathrm{Bi}=0.1, \mathrm{Ra}=10^{5}\right)$. The times are given in dimensional units for ease of comparison and indicative colour scales are given on the right. 
forces are small, as are the velocities. There is also a large region of relatively cold gas, in fact close to the initial temperature, near the bottom of the reactor. As the reaction proceeds, the hot spot in the reactor shifts further up the vertical axis and eventually the explosion initiates in this hot region. There is evidence for all times shown in Fig. 3(a) of a thin thermal boundary layer at the top of the vessel which remains close to the initial temperature. This is despite the fact that the wall temperature is no longer held constant due to the existence of external heat transfer.

Shown in Fig. 3(b) is case F, where $\mathrm{Bi}=10$ and $\mathrm{Ra}=10^{5}$. It can be seen that the temperature in the vessel increases relatively uniformly over the first second of reaction, and thereafter a hot zone forms close to the top of the vessel. This hot zone can be seen to develop for $t>2 \mathrm{~s}$. As the convection is much more intense than that shown in Fig. 3(a), the hot zone is much closer to the top of the reactor. Additionally, the familiar horizontal stratification of the temperature, which is characteristic of cases when natural convection is significant, is evident. Due to the more intense convection and the proximity of the hot zone to the top of the reactor, the thermal boundary layer at a temperature close to the initial temperature, as seen in Fig. 3(a) for all times, persists only for the first $3 \mathrm{~s}$ of reaction. After that, it is evident that the intense natural convection, coupled to the removal of heat externally leads to the wall of the vessel heating up. The speed profiles show that as the vessel warms uniformly at the beginning of the reaction, there is significant upflow around the centreline of the reactor, and the warm gas descending along the cold walls moves very rapidly. Indeed, Fig. 3(b) shows that the flow in the thin layer close to the wall is very fast compared to the central up flow for $t>2 \mathrm{~s}$. These temperature and velocity fields are structurally very similar to those presented previously ${ }^{10}$ for explosive cases with a constant wall temperature. This is perhaps unsurprising given that when $\mathrm{Bi}=10$, the internal heat transfer will still largely control the overall heat transfer. As mentioned above, the principal difference between Fig. 3(a) and (b) and their constant wall temperature counterparts is the erosion of the thermal boundary layer near the top of the vessel, due to the variation in the wall temperature that accompanies the external heat loss.

If the heat transfer coefficient describing heat loss from the external wall is decreased, then Bi will also decrease. Shown in Fig. $3(\mathrm{c})$ is the case where $\mathrm{Bi}=1$ and $\mathrm{Ra}=10^{3}$ i.e. the internal and external heat transfer resistances are of similar order and natural convection is just becoming significant. The temporal development of the temperature shows a similar pattern to that in Fig. 3(a), with the temperature increasing approximately uniformly over the entire cross section of the reactor over the first 2 seconds of reaction before a hot zone develops above the centre of the reactor due to the effects of the convection. The location of this hot zone is similar to that in Fig. 3(a), which is to be expected given the similar intensities of natural convection. The shape of the temperature contours is also qualitatively similar in the two cases. Where Fig. 3(a) and (c) differ though is in the magnitude of the temperature gradients. Whilst the case with $\mathrm{Bi}=10$ in Fig. 3(a) shows a cold zone, with a temperature close to its initial value, toward the bottom of the reactor and around the wall, when $\mathrm{Bi}$ is decreased in Fig. 3(c) then these regions show significant warming. There is therefore still spatial variation in the temperature in this case, but the gradients are considerably smaller. If the development of the velocity field is examined there is again some similarity to what is seen in Fig. 3(a) i.e. there is an area of higher speed that develops in the centre of the reactor after approximately $1 \mathrm{~s}$ of reaction and a region of higher speed flow near the wall. It is interesting to note that this downflow close to the wall takes longer to develop in this case than it does for the higher Bi case. This is most likely due to the fact that the wall temperature is much larger in case $\mathrm{H}$ in Fig. 3(c) than in case B in Fig. 3(a) and therefore the descending gas is cooled less and so is less dense than in case B and hence there is less of a buoyancy driving force for downflow.

Fig. 3(d) shows the development of temperature and speed for case $\mathrm{L}$, where $\mathrm{Bi}=1$ and $\mathrm{Ra}=10^{5}$. It can be seen from Fig. $3(\mathrm{~d})$ that the temperature increases virtually uniformly over the entire cross section of the reactor for the first $3 \mathrm{~s}$, with only a small cold zone existing toward the bottom of the reactor. Subsequently, the hot zone begins to develop close to the top of the reactor. Its location is very similar to that seen in Fig. 3 (b) for $\mathrm{Bi}=10$, but as was the case for the low $\mathrm{Ra}$ system at $\mathrm{Bi}=1$, the variation in temperature throughout the reactor is much less than in the higher Bi cases. Thus, the hot zone in Fig. 3(d) (e.g. at $t=12 \mathrm{~s})$ is much larger than that seen in Fig. 3(b) (e.g. at $t=6 \mathrm{~s}$ ). The development of the speed in Fig. 3(d) in comparison to that in Fig. 3(b) is also interesting. In both cases after $\sim 1 \mathrm{~s}$ a region of relatively strong upflow develops about the centreline. Subsequently, a region of faster downflow along the wall in the upper half of the vessel emerges. It is interesting to note that the extent of this region is much less in the case of $\mathrm{Bi}=1$ in Fig. 3(d) than when $\mathrm{Bi}=10$ in Fig. 3(b). As discussed above, this is most likely due to the higher wall temperature that exists in the lower Bi case. As the flow field develops in Fig. 3(d) it is clear that flow near the vertical axis in the top half of the vessel is more significant than was the case for $\mathrm{Bi}=10$.

Fig. 3(e) shows case $\mathrm{N}$ where $\mathrm{Bi}=0.1$ and $\mathrm{Ra}=10^{3}$. When $\mathrm{Bi} \ll 1$, the temperature within the system is expected to be approximately spatially uniform. ${ }^{29}$ It is evident from Fig. 3(e) that this is indeed the case. Virtually no gradients in the temperature are visible. The development of the speed is similar to the previous cases with $\mathrm{Ra}=10^{3}$ in that there is a higher speed region around the centreline. It can be seen that this region is not however shifted towards the top of the reactor as is the case in Fig. 3(a) and (c). This is obviously because there is not a hot zone formed above the centre of the reactor, rather the temperature is uniform over the whole cross section. In Fig. 3(f) is plotted case $\mathrm{R}$ with $\mathrm{Bi}=0.1$ and $\mathrm{Ra}=10^{5}$. As in the low Ra case, the temperature can be seen to develop similarly across the entire cross section. The velocity field is established in the first second of reaction and then changes very little. The intense mixing of course contributes to the spatial uniformity in temperature.

\subsection{The effect of $\mathrm{Bi}$ on the maximum temperature in a stable system}

It was shown above that the primary difference between the temporal developments of the maximum temperature in stable 
systems as $\mathrm{Bi}$ varied was the final temperatures reached, with lower temperatures occurring for lower values of $\mathrm{Bi}$. There also seemed to be little difference between the cases at fixed $\mathrm{Bi}$ as $\mathrm{Ra}$ varied. To elucidate further these effects, simulations were performed to capture the maximum temperature that occurs in the steady state for a stable solution on the explosion boundary (such as is shown in Fig. 1). This was done for a range of $\mathrm{Bi}$ and at three values of $\mathrm{Ra}$, i.e. $10^{3}, 10^{4}$ and $10^{5}$. The results are plotted in Fig. 4 . When $\mathrm{Bi}=0$, the system is perfectly mixed and as predicted by Semenov, ${ }^{1}$ the maximum dimensionless temperature of a stable system is 1 . When $\mathrm{Bi}$ is increased, it is clear that there is an increase in the maximum temperature that can be achieved before explosion occurs. Indeed, for $\mathrm{Bi}>5$, for the range of $\mathrm{Ra}$ considered, it can be seen that the maximum temperature rises above the value of 1.6 that was predicted by Frank-Kamenetskii ${ }^{2}$ for a purely diffusive system. For each $\mathrm{Ra}$ when $\mathrm{Bi}<\sim 10$ there is very little difference in the maximum temperatures achievable in a nonexplosive case. What is interesting to note, however, is that the maximum temperature for each $\mathrm{Bi}$ in this range is actually seen for the lowest value of Ra. This result is somewhat counterintuitive and will be discussed further below. Of course, it should be borne in mind that these maximum temperatures were derived numerically and the maximum temperature is sensitive to the proximity to the explosion boundary, nevertheless the trend is evident for all $\mathrm{Bi}<10$. This trend is reversed as $\mathrm{Bi}$ increases above 20, where the increased intensity of convection indicated by a larger $\mathrm{Ra}$ results in a higher temperature being achieved in a stable reaction. When Bi increases above 25, the maximum temperatures achieved diverge and the effect of the more intense convection is obvious. The maximum temperatures then clearly approach the values achieved in a constant wall temperature case (i.e. $\mathrm{Bi}=\infty$ ) asymptotically. Thus, for $\mathrm{Ra}=10^{3}$, the maximum temperature is approximately 2.5 , for $\mathrm{Ra}=10^{4}$ it is 3.15 and for $\mathrm{Ra}=10^{5}$ it is 3.45. Unsurprisingly, all three values are comfortably above the value of 1.6 suggested for diffusive systems, ${ }^{2}$ with a higher temperature being possible in a

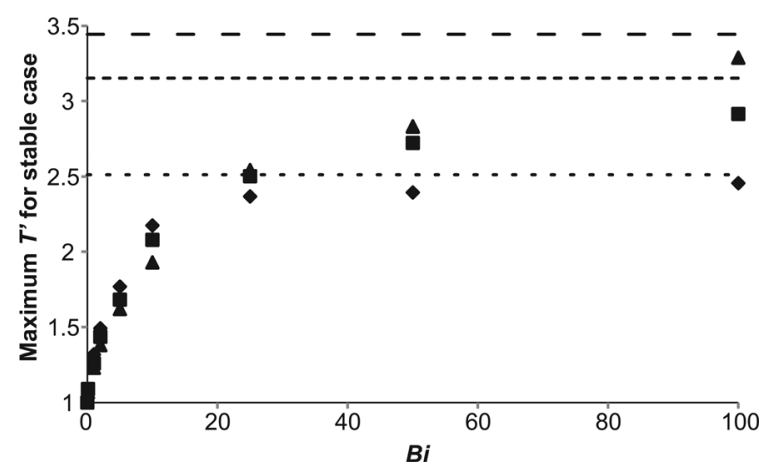

Fig. 4 The maximum temperature in the reactor at steady state found numerically for a stable system on the explosion boundary as a function of $\mathrm{Bi}$ and $\mathrm{Ra}$. The diamonds are for cases with $\mathrm{Ra}=10^{3}$, the squares represent $\mathrm{Ra}=10^{4}$ and the triangles are $\mathrm{Ra}=10^{5}$. Also plotted are horizontal lines which show the maximum temperatures for non-explosive cases with a constant wall temperature (i.e. $\mathrm{Bi}=\infty$ ). The dotted line is for $\mathrm{Ra}=10^{3}$, the dashed line for $\mathrm{Ra}=10^{4}$ and the open dashed line for $\mathrm{Ra}=10^{5}$. system with strong convection, due to the improved heat removal. Interestingly, all three values are below the $T^{\prime}=5$ threshold suggested $^{11}$ as a criterion for explosion in systems with considerable consumption of reactant. The most important consequence of the variation of the maximum temperature in a stable system with $\mathrm{Bi}$ is that a simple criterion for explosion, based on a constant value of dimensionless temperature, may be inappropriate for cases with external heat transfer. This is a topic which clearly merits further study, when systems with significant consumption of reactant are investigated.

As remarked upon above, there is a shift in the relative values of the maximum dimensionless temperature for stable cases with fixed Ra from the temperatures being very similar, but with the maximum occurring at the lowest value of Ra considered, at low $\mathrm{Bi}$ to those with the maximum temperature being observed for the highest value of $\mathrm{Ra}$ when $\mathrm{Bi}>\sim 20$. To investigate this further, nine simulations were performed for cases very close to the explosion boundary, but in the stable region, for $\mathrm{Bi}=1,10$ and 100 with $\mathrm{Ra}=10^{3}, 10^{4}$ and $10^{5}$. A summary of the conditions used for these simulations appears in Table 2. Shown in Fig. 5 are the temperature profiles along the vertical axis and around the surface of the vessel at steady state for each of the nine cases considered. For $\mathrm{Bi}=100$, it can be seen that the vertical profiles are very similar to those seen, both numerically ${ }^{19}$ and experimentally, ${ }^{5}$ when the wall is held at a fixed temperature. The bottom surface of the reactor remains close to its original temperature, and a skewed temperature profile develops, with the point of maximum temperature occurring well above the centre of the reactor. Indeed, as Ra increases and the convection becomes more intense, the point of maximum shifts closer to the top of the vessel, and the increased heat removal leads to a higher temperature in the hot zone. It can also be seen that the difference in temperature between the hot zone and the wall, which is the driving force for heat transfer, is largest for the highest $\mathrm{Ra}$. When considering the surface temperature it can be seen that there is significant variation in temperature around the vertical circumference of the reactor. This is despite the fact that $\mathrm{Bi}=100$ implies that internal heat transfer is dominant. As one would expect, the temperature of the surface increases monotonically from the bottom towards the top, with the highest temperature occurring at the very top of the reactor.

Table 2 Summary of the conditions for the simulations presented in Fig. 5 and 6 for stable cases on the explosion boundary with $\mathrm{Bi}=1,10$ or 100 and $\mathrm{Ra}=10^{3}, 10^{4}$ or $10^{5}$

\begin{tabular}{llll}
\hline$\frac{\tau_{\mathrm{H}}}{\tau_{\mathrm{C}}}$ & $\frac{\tau_{\mathrm{H}}}{\tau_{\mathrm{D}}}$ & $\mathrm{Bi}$ & $\mathrm{Ra}$ \\
\hline 6.8 & 0.215 & 100 & $10^{3}$ \\
13.7 & 0.137 & & $10^{4}$ \\
26.75 & 0.0846 & & $10^{5}$ \\
9.1 & 0.288 & 10 & $10^{3}$ \\
21.85 & 0.219 & & $10^{4}$ \\
53.95 & 0.171 & & $10^{5}$ \\
33.75 & & 1 & $10^{3}$ \\
102.35 & 1.067 & & $10^{4}$ \\
310.9 & 1.024 & & $10^{5}$
\end{tabular}



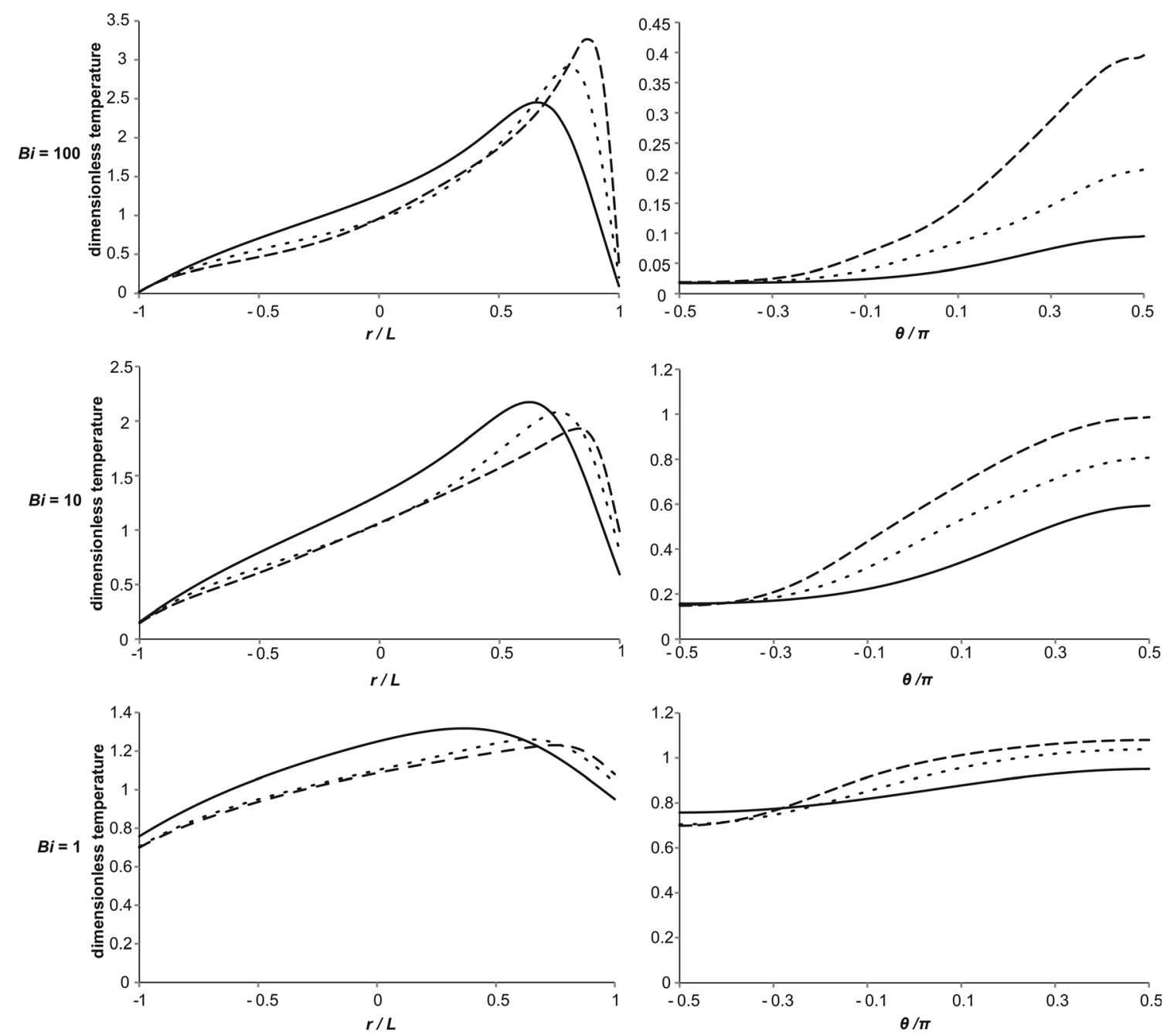

Fig. 5 The variation in the vertical temperature profile (left column) and the surface temperature (right column) at steady state for stable reactions very close to the explosion boundary. For the vertical profiles, the origin is taken as the centre of the vessel, and the location on the surface of the vessel is represented by the positive angle $(\theta)$, measured anti-clockwise from the horizontal axis of the reactor. The conditions used are summarised in Table 2 . The horizontal pairs correspond to simulations at constant $\mathrm{Bi}$, with values taken as 100,10 or 1 . In each case the solid line represents $\mathrm{Ra}=10^{3}$, the dotted line $\mathrm{Ra}=10^{4}$ and the dashed line $\mathrm{Ra}=10^{5}$.

There is very little variation in the surface temperature in the bottom half of the reactor, whereas the hot gas that has passed through the hot zone and cools as it descends along the wall causes a more significant increase in the temperature in the top half of the vessel. Clearly also the more intense the convection, the more heat is transferred to the surface and hence the hotter the surface becomes. It should also be noted that although there is significant variation in the surface temperature upon moving from the bottom to the top of the reactor, the maximum dimensionless surface temperature is an order of magnitude smaller than that in the hot zone of the reactor.

When considering the cases with $\mathrm{Bi}=10$, it is evident that there are many qualitative similarities between the vertical and surface temperature profiles and their counterparts for $\mathrm{Bi}=100$. Considering first the vertical profile, the lowest temperature can be seen at the bottom of the reactor and the hot spot is located above the centre and shifts upwards with Ra. Indeed, it can be seen that the location of the hot spot for each Ra is in a very similar location to those when $\mathrm{Bi}=100$. When comparing the cases for $\mathrm{Bi}=10$ and $\mathrm{Bi}=100$ it is obvious from Fig. 5 that the temperature of the bottom of the reactor is increased only very slightly when $\mathrm{Bi}$ is reduced from 100 to 10 . There is clearly a much more significant increase in the temperature at the top surface of the reactor for $B i=10$. Additionally, when $B i=10$, as described above, the maximum temperature that is achieved in a stable system decreases with increasing Ra, although the values are much closer together than is the case when $\mathrm{Bi}=100$.

When $\mathrm{Bi}$ is reduced to 1 then internal and external resistances to heat transfer are of similar order. This has the clear effect of making the temperature both within, and on the surface of the reactor much more uniform. The skewed vertical profile is still evident, but the gradients are clearly much smaller, due to the higher surface temperatures. The reduction in temperature difference between the hot zone and the vessel walls means that there is a lower driving force for heat transfer and hence lower temperatures than those seen in the high 


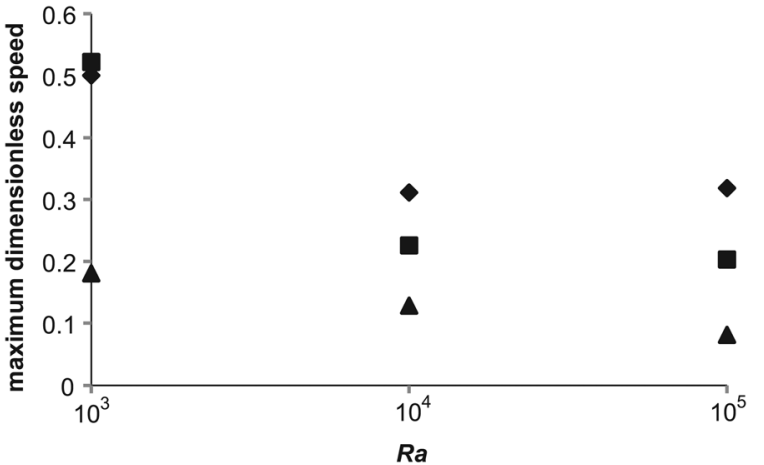

Fig. 6 The maximum dimensionless speed at steady state for the nine stable cases, close to the explosion boundary, described in Table 2. The diamonds represent those cases with $\mathrm{Bi}=100$, the squares are cases with $\mathrm{Bi}=10$ and the triangles are those with $\mathrm{Bi}=1$.

Bi cases can be sustained without explosion. Whilst the spatial location of the hot spot in the reactor as a function of Ra is very similar for $\mathrm{Bi}=100$ and 10 , the hot zone occurs lower down the vertical axis when $B i=1$, indicating the reduced significance of the flow due to natural convection. Once again, the maximum temperatures on the vertical axis decrease with increased Ra as do the temperature differences between the hot zone and the top surface. When considering the surface temperature, it is clear that the most significant change upon decreasing Bi from 10 to 1 is in the temperature distribution in the bottom half of the reactor. The surface temperatures in the top half change comparatively little, whereas those in the bottom half increase markedly, to give a more uniform profile.

To further investigate the effect of varying Bi on the intensity of convection, and hence the ability to remove heat from the system, the maximum dimensionless speed in each of the nine cases described in Table 2 was plotted as a function of Ra in Fig. 6. For each $\mathrm{Bi}$ it is clear that the value of the dimensionless speed is the largest when $\mathrm{Ra}=1000$; however, it should be noted that this is most likely due to the fact that the scale used for velocity assumes that convection is dominant, whereas for $\mathrm{Ra}=1000$, diffusive processes will still play a significant role. For the larger values of Ra it is obvious that the maximum dimensionless speed in the reactor at steady state decreases as $\mathrm{Bi}$ increases. For the case with $\mathrm{Bi}=10$ and 1, the maximum speed decreases slightly as Ra is increased from $10^{4}$ to $10^{5}$. This is in contrast to the $\mathrm{Bi}=100$ cases where the maximum speed increases very slightly upon a similar increase in Ra. These observations may indicate that Ra as defined in eqn (17) does not give a true reflection of the intensity of convection in cases where the wall temperature is changing (i.e. cases with smaller Bi). This may also go some of the way to explaining the trends in maximum temperature (i.e. the maximum temperature of a stable system decreases with Ra for low Bi and increases with $\mathrm{Ra}$ at high Bi) discussed above.

\subsection{Identification of explosive conditions as Bi varies}

As has been shown above, the variation of external heat transfer, characterised by $\mathrm{Bi}$, can have a significant effect on the behaviour of an exothermic reaction under the influence of

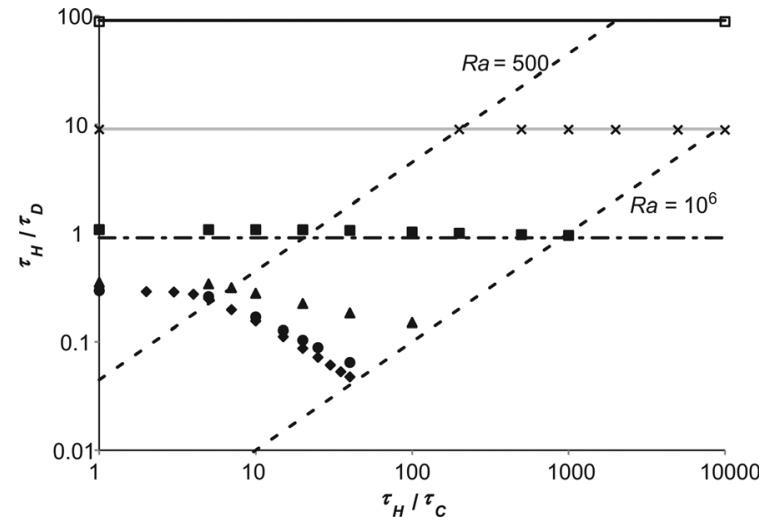

Fig. 7 The regime diagram for explosion showing the numerically derived explosion limits (for $\mathrm{Ra}<10^{6}$ ) for a range of Bi. Also shown are two dashed lines which show constant values of $\mathrm{Ra}$. The diamonds show the limit for $B i=\infty$, the circles show $B i=100$, the triangles $B i=10$, the filled squares $\mathrm{Bi}=1$, the crosses $\mathrm{Bi}=0.1$ and the open squares $\mathrm{Bi}=0.01$. The horizontal lines show Semenov's prediction, via eqn (20), for the explosive limit in a well-mixed system with varying $\mathrm{Bi}$. The dot-dash line shows eqn (20) for $\mathrm{Bi}=1$, the grey line $\mathrm{Bi}=0.1$ and the black line $\mathrm{Bi}=0.01$.

natural convection. Of course, one of the most important questions that must be addressed is what effect does this external heat transfer resistance have on the tendency of a system to explode? To investigate this, many simulations were performed to find the boundaries of the explosive region for different values of Bi. These limits are summarised in Fig. 7, which shows a regime diagram, such as presented in Fig. 1, plotted on logarithmic scales. The boundary for $\mathrm{Bi}=\infty$ is the same as that shown in Fig. 1, and agrees well with previous studies. ${ }^{10}$ The region below the boundary is where explosions occur, and that above it represents stable reactions. Two regions of behaviour are shown very clearly in Fig. 7. For Ra $<500$, diffusion dominates and the explosion limit is approximately constant. For $\mathrm{Ra}>\sim 1000$, then natural convection is important and there is clearly a power law variation in the explosion limit of the form

$$
\left(\frac{\tau_{\mathrm{H}}}{\tau_{\mathrm{D}}}\right)_{\text {explosion }} \sim\left(\frac{\tau_{\mathrm{H}}}{\tau_{\mathrm{C}}}\right)^{\alpha}
$$

where Liu et al..$^{10,11}$ found this exponent to be -0.74 for $500<$ $\mathrm{Ra}<10^{6}$. The value found in this work is -0.82 , which agrees reasonably well given the precision with which the boundary was found previously. Simulations for Ra $>10^{6}$ (i.e. turbulent flows) have not been considered here, although the value of the exponent $\alpha$ has been predicted analytically ${ }^{10}$ to be -2 .

When a small amount of external heat transfer is allowed for (i.e. when $\mathrm{Bi}=100$ ), it can be seen that for $\mathrm{Ra}<500$, the explosion limit is virtually identical to that for the constant wall temperature case. As Ra increases above 500 and natural convection becomes more significant, it can be seen that explosions occur at a slightly higher value of $\tau_{\mathrm{H}} / \tau_{\mathrm{D}}$ than when $\mathrm{Bi}=\infty$. This is of course due to the fact that the external resistance to heat transfer lessens somewhat the stabilising effect of the increased fluid flow and hence heat transfer from the contents of the vessel 


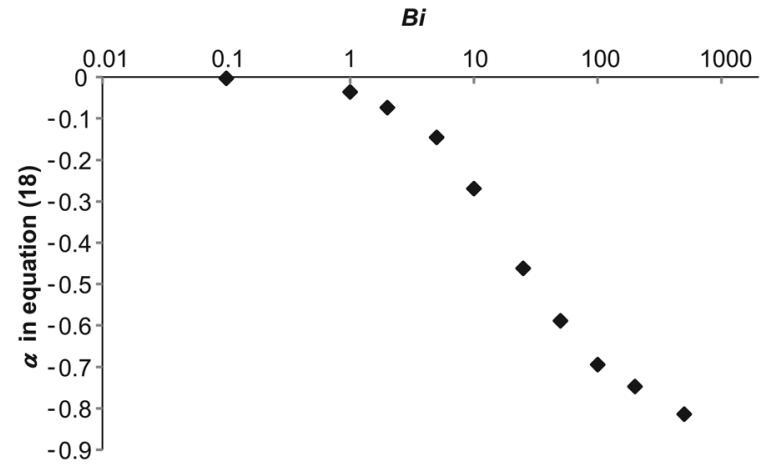

Fig. 8 The exponent in eqn (18), which characterises the stabilising effect of natural convection on the system, as a function of $\mathrm{Bi}$.

to the wall. It is also evident that the power law behaviour described in eqn (18) still exists, though the value of the exponent is reduced.

For $\mathrm{Bi}=10$ it is shown in Fig. 7 that the critical value of $\tau_{\mathrm{H}} / \tau_{\mathrm{D}}$ in the diffusive region is increased slightly and that the stabilising effect of convection has been substantially lessened. For $B i=1$, there is still a very slight stabilising effect of convection evident for $500<\mathrm{Ra}<10^{6}$, however for $\mathrm{Bi}<1$, the explosive limit has an approximately constant value of $\tau_{\mathrm{H}} / \tau_{\mathrm{D}}$. It is shown very clearly in Fig. 7 that external heat transfer has two main effects $v i z$. it increases the critical value of $\tau_{\mathrm{H}} / \tau_{\mathrm{D}}$ indicating that the system is more prone to explode and it also lessens the stabilising effect of natural convection.

The extent to which the stabilising effect of convection is suppressed can be characterised by the exponent in eqn (18). The variation of this exponent is shown, as derived from a bestfit of the numerical results, in Fig. 8. Clearly, when Bi is very small, the explosive limit is approximately constant so $\alpha$ is close to zero. The bulk of the variation in $\alpha$ is seen over the range $1<\mathrm{Bi}<100$, which is similar to the range reported for main effect of $\mathrm{Bi}$ on explosion in diffusive systems with consumption of reactant. ${ }^{16}$ For $\mathrm{Bi}>100$, the value of the exponent in eqn (18) approaches the $\mathrm{Bi}=\infty$ value asymptotically.

\subsection{The Frank-Kamenetskii and Semenov limits}

The numerically derived explosion limits presented in Fig. 8 can be compared to analytical solutions in the FrankKamenetskii and Semenov limits. Frank-Kamenteskii derived ${ }^{2}$ an approximate expression for the transition to explosion which can be written as

$$
\left(\frac{\tau_{\mathrm{H}}}{\tau_{\mathrm{D}}}\right)_{\text {explosion, } \mathrm{FK}}=\left(\frac{\tau_{\mathrm{H}}}{\tau_{\mathrm{D}}}\right)_{\mathrm{Bi}=0}\left(1+\frac{e}{\mathrm{Bi}}\right)
$$

Plotted in Fig. 9 are the numerically derived explosive limits for a purely diffusive system and a line representing eqn (19). There is clearly an excellent agreement between Frank-Kamenetskii's prediction and the numerical results. As observed above, the value of $\tau_{\mathrm{H}} / \tau_{\mathrm{D}}$ at the explosive limit is approximately constant for $\mathrm{Bi}>10$ and as predicted by eqn (19) $\tau_{\mathrm{H}} / \tau_{\mathrm{D}}$ is proportional to $\mathrm{Bi}^{-1}$ as $\mathrm{Bi}$ tends to zero and hence $1+e / \mathrm{Bi}$ tends to $e / \mathrm{Bi}$.

At small values of $\mathrm{Bi}$, where external heat transfer is the dominant mechanism, the Semenov limit of a well-mixed

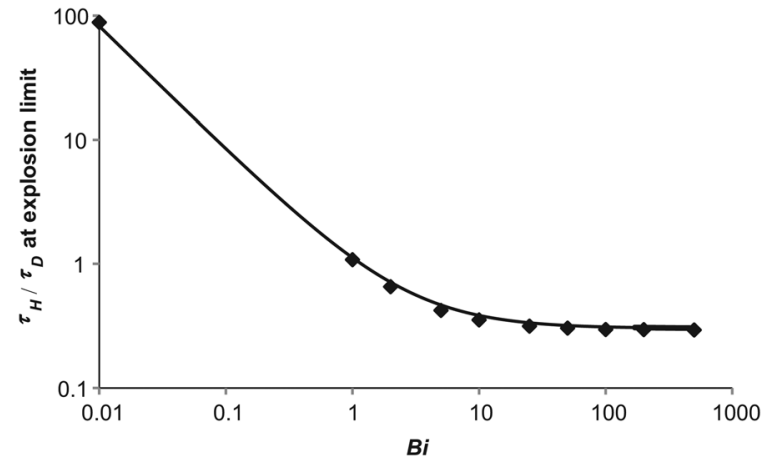

Fig. 9 A comparison of the numerically derived explosion limit for a purely diffusive system for different values of Bi with Frank-Kamenetskii's approximate expression in eqn (19). The diamonds are the numerically derived limits and the solid line represents eqn (19).

system is approached. Eqn (1) can be re-written in terms of the characteristic timescales used here as

$$
\left(\frac{\tau_{\mathrm{H}}}{\tau_{\mathrm{D}}}\right)_{\text {explosion,Semenov }}=\frac{e}{3 \mathrm{Bi}}
$$

Eqn (20) is plotted for $B i=0.01,0.1$ and 1 in Fig. 7. For $B i=1$, eqn (20) slightly under predicts the explosive limit and, of course, does not capture the marginal stabilising effect of convection. This discrepancy is unsurprising given that there is still some spatial variation in the temperature field, as shown in Fig. 5. For $\mathrm{Bi} \ll 1$, the agreement between eqn (20) and the numerically derived boundaries can be seen to be excellent in Fig. 7, with the numerically derived results agreeing with Semenov's prediction to within $2 \%$. This excellent agreement between the analytical predictions in both limits not only provides further validation of the numerical methods used, but also shows that the inclusion of external heat transfer in systems with natural convection truly bridges these two classical limits.

\section{Conclusions}

The behaviour of an exothermic chemical reaction occurring in a spherical vessel under the influence of natural convection and an external heat transfer resistance, but without consumption of reactant, has been studied numerically. It has been shown that the external heat transfer, as characterised by a Biot number, has very little qualitative impact on the temporal development of the maximum temperature in the reactor during explosive and non-explosive reactions. The main quantitative effect is that the reactions take place at a lower temperature when $\mathrm{Bi}$ is reduced. Indeed, it has been shown that the maximum dimensionless temperature achieved in a non-explosive reaction on the explosion boundary increases from 1 in the well mixed limit to its constant wall temperature value asymptotically. For $\mathrm{Bi}<\sim 10$, the maximum temperature varies little with Rayleigh number, but interestingly is lower for higher values of Ra. This is in contrast to what is observed at higher $\mathrm{Bi}$ where more intense natural convection means that higher temperatures can be sustained in non-explosive cases. This effect may be due to the reduction in 
driving force for heat transfer from the hot zone of the reactor to the wall. This is primarily due to the fact that the wall temperature increases much more significantly as Bi is reduced. Importantly, this variation in maximum temperature achieved in stable systems means that explosion criteria based on the maximum temperature achieved in the reactor may be inappropriate for systems with significant external heat transfer.

A region of parameter space where explosions occur has been defined for a series of values of $\mathrm{Bi}$. It has clearly been shown that the inclusion of external heat transfer means that the region of parameter space where explosions occur is larger and that the stabilising effect of natural convection on the reaction is greatly diminished as $\mathrm{Bi}$ is reduced, particularly over the range 100-1. Finally, the numerical results have been shown to agree very well with the analytical solutions of Frank-Kamenetskii and Semenov in the purely diffusive and well-mixed limits.

\section{Nomenclature}

\begin{tabular}{|c|c|}
\hline $\mathrm{Bi}$ & Biot number, $\mathrm{Bi}=h L / k_{\mathrm{T}}$ \\
\hline$c$ & concentration of reactant \\
\hline$c^{\prime}$ & dimensionless concentration of reactant, $c^{\prime}=c / c_{0}$ \\
\hline$c_{0}$ & initial concentration of reactant \\
\hline$C_{\mathrm{P}}$ & specific heat at constant pressure \\
\hline$D_{\mathrm{C}}$ & diffusion coefficient of reactant \\
\hline E & activation energy \\
\hline$g$ & acceleration due to gravity \\
\hline$h$ & external heat transfer coefficient \\
\hline$k$ & rate constant \\
\hline$k_{0}$ & rate constant evaluated at $T=T_{0}$ \\
\hline$k_{\mathrm{T}}$ & thermal conductivity \\
\hline$L$ & characteristic length (radius) of the reactor \\
\hline Le & Lewis number, Le $=\kappa / D_{C}$ \\
\hline$n$ & order of the reaction \\
\hline$P$ & pressure \\
\hline$P_{0}$ & initial pressure \\
\hline$P^{\prime}$ & dimensionless pressure, $P^{\prime}=\left(P-P_{0}\right) / \rho_{0} U^{2}$ \\
\hline $\operatorname{Pr}$ & Prandtl number, $\operatorname{Pr}=\nu / \kappa$ \\
\hline$q$ & exothermicity of the reaction \\
\hline$r$ & radial coordinate \\
\hline$R$ & universal gas constant \\
\hline $\mathrm{Ra}$ & Rayleigh number, $\mathrm{Ra}=\beta g L^{3} \Delta T / \kappa \nu$ \\
\hline$S_{\mathrm{v}}$ & surface area per unit volume \\
\hline$t$ & time \\
\hline$t^{\prime}$ & dimensionless time, $t^{\prime}=U t / L$ \\
\hline$T$ & temperature \\
\hline$T^{\prime}$ & $\begin{array}{l}\text { dimensionless rise in temperature, } \\
T^{\prime}=E\left(T-T_{0}\right) / R T_{0}^{2}\end{array}$ \\
\hline$T_{\text {ad }}{ }^{\prime}$ & $\begin{array}{l}\text { dimensionless adiabatic temperature increase, } \\
T_{\mathrm{ad}}{ }^{\prime}=q c_{0} E /\left(\rho_{0} C_{\mathrm{P}} R T_{0}{ }^{2}\right)\end{array}$ \\
\hline$T_{0}$ & constant temperature of the environment \\
\hline$\underline{u}$ & velocity \\
\hline$\underline{u}^{\prime}$ & dimensionless velocity, $\underline{u}^{\prime}=\underline{u} / U$ \\
\hline$U$ & characteristic velocity \\
\hline$x$ & spatial coordinates \\
\hline
\end{tabular}

$\begin{array}{ll}x^{\prime} & \text { dimensionless spatial coordinates, } x^{\prime}=x / L \\ Z & \text { pre-exponential factor in the Arrhenius term } \\ \beta & \text { coefficient of thermal expansion } \\ \delta & \text { Frank-Kamenetskii parameter, } \delta=L^{2} k_{0} c_{0}^{n} q E / \kappa \rho_{0} C_{\mathrm{P}} R T_{0}{ }^{2} \\ \Delta T & \text { characteristic temperature increase, } \Delta T=R T_{0}{ }^{2} / E \\ \eta & \text { dimensionless parameter, } \eta=R T_{0} / E \\ \theta & \text { angle measured anti-clockwise from the horizontal } \\ \kappa & \text { axis of the reactor } \\ \mu & \text { thermal diffusivity } \\ \nu & \text { viscosity } \\ \rho & \text { kinematic viscosity } \\ \rho_{0} & \text { density } \\ \tau_{\mathrm{C}} & \text { density at } T=T_{0} \\ \tau_{\mathrm{D}} & \text { timescale for convection } \\ \tau_{\mathrm{H}} & \text { timescale for diffusion of heat } \\ \psi & \text { timescale for heating by reaction } \\ & \text { Semenov number, } \psi=k_{0} c_{0}^{n} q E / h S_{\mathrm{v}} R T_{0}{ }^{2}\end{array}$

\section{Acknowledgements}

The financial support of the Engineering and Physical Sciences Research Council via Small Equipment Grant EP/K031317/1 is gratefully acknowledged.

\section{References}

1 N. N. Semenov, Z. Phys., 1928, 48, 571-582.

2 D. A. Frank-Kamenetskii, in Diffusion and Heat Transfer in Chemical Kinetics, ed. J. P. Appleton, Plenum, 2nd edn, 1969.

3 B. J. Tyler, Combust. Flame, 1966, 10, 90-91.

4 P. G. Ashmore, B. J. Tyler, T. A. B. Wesley, Eleventh Symposium (International) on Combustion, 1967, 1133-1140.

$5 \mathrm{~W}$. H. Archer, PhD thesis, University of Manchester Institute of Science and Technology, 1977.

6 A. G. Merzhanov and E. A. Shtessel, Combust., Explos. Shock Waves (Engl. Transl.), 1971, 7, 58-65.

7 D. R. Jones, Int. J. Heat Mass Transfer, 1973, 16, 157-167.

8 E. A. Shtessel, K. V. Priytkova and A. G. Merzhanov, Combust., Explos. Shock Waves (Engl. Transl.), 1971, 7, 137-146.

9 A. G. Merzhanov and E. A. Shtessel, Astronaut. Acta, 1973, 18, 191-199.

10 T.-Y. Liu, A. N. Campbell, S. S. S. Cardoso and A. N. Hayhurst, Phys. Chem. Chem. Phys., 2008, 10, 5521-5530.

11 T.-Y. Liu, A. N. Campbell, A. N. Hayhurst and S. S. S. Cardoso, Combust. Flame, 2010, 157, 230-239.

12 F. G. de Azevedo, J. F. Griffiths and S. S. S. Cardoso, Combust. Flame, 2014, 161, 1752-1755.

13 F. G. de Azevedo, J. F. Griffiths and S. S. S. Cardoso, Phys. Chem. Chem. Phys., 2013, 15, 16713-16724.

14 T.-Y. Liu and S. S. S. Cardoso, Combust. Flame, 2013, 160, 191-203.

15 F. G. de Azevedo, J. F. Griffiths and S. S. S. Cardoso, Phys. Chem. Chem. Phys., 2014, 16, 23365-23378. 
16 T. Boddington, C. Feng and P. Gray, Proc. R. Soc. A, 1984, 391, 269-294.

17 J. S. Turner, Buoyancy Effects in Fluids, Cambridge University Press, 1979, pp. 207-250.

18 T.-Y. Liu and S. S. S. Cardoso, Phys. Chem. Chem. Phys., 2012, 14, 192-199.

19 A. N. Campbell, S. S. S. Cardoso and A. N. Hayhurst, Chem. Eng. Sci., 2007, 62, 3068-3082.

20 T. Dumont, S. Ge'nieys, M. Massot and V. A. Volpert, SIAM J. Appl. Math., 2002, 63, 351-372.

21 S. S. S. Cardoso, P. C. Kan, K. K. Savjani, A. N. Hayhurst and J. F. Griffiths, Combust. Flame, 2004, 136, 241-245.

22 S. S. S. Cardoso, P. C. Kan, K. K. Savjani, A. N. Hayhurst and J. F. Griffiths, Phys. Chem. Chem. Phys., 2004, 6, 1687-1696.

23 A. N. Campbell, S. S. S. Cardoso and A. N. Hayhurst, Proc. $R$. Soc. A, 2005, 461, 1999-2020.
24 A. N. Campbell, S. S. S. Cardoso and A. N. Hayhurst, Chem. Eng. Res. Des., 2006, 84, 553-561.

25 A. N. Campbell, S. S. S. Cardoso and A. N. Hayhurst, Chem. Eng. Sci., 2005, 60, 5705-5717.

26 A. N. Campbell, S. S. S. Cardoso and A. N. Hayhurst, Combust. Flame, 2008, 154, 122-142.

27 M. R. Foster and H. Pearlman, Combust. Flame, 2008, 147, 108-117.

28 M. R. Foster and H. Pearlman, Microgravity Sci. Technol., 2012, 24, 113-125.

29 F. P. Incropera and D. P. DeWitt, Fundamentals of Heat and Mass Transfer, Wiley, 4th edn, 1996, pp. 215-216.

30 COMSOL, COMSOL Multiphysics Reference Guide Version 4.3a, 2012.

31 A. N. Campbell, S. S. S. Cardoso and A. N. Hayhurst, Phys. Chem. Chem. Phys., 2006, 8, 2866-2878. 